\title{
Assessment of cardiac autonomic functions by heart rate recovery indices in patients with myocardial bridge
}

Sercan Okutucu ${ }^{1}$, Mustafa Aparci ${ }^{2}$, Cengiz Sabanoglu ${ }^{3}$, Ugur Nadir Karakulak ${ }^{4}$, Hakan Aksoy ${ }^{1}$, Cengiz Ozturk ${ }^{5}$, Mehmet Karaduman ${ }^{6}, Z_{\text {Zafer Isilak }}^{2}$, Adem Adar ${ }^{7}$, Ali Oto ${ }^{1}$

${ }^{1}$ Department of Cardiology, Memorial Ankara Hospital, Ankara, Turkey.

${ }^{2}$ Department of Cardiology, Haydarpasa Training Hospital, Istanbul, Turkey ${ }^{3}$ Department of Cardiology, Kirikkale High Speciality Hospital, Kirikkale, Turkey

${ }^{4}$ Department of Cardiology, Occupational Diseases Hospital, Ankara Turkey

${ }^{5}$ Department of Cardiology, Gulhane Military Medical Faculty, Ankara, Turkey

${ }^{6}$ Department of Internal Medicine, Gulhane Military Medical Faculty, Ankara, Turkey

${ }^{7}$ Department of Cardiology, Karabuk State Hospital, Karabuk, Turkey

\begin{abstract}
Background: Heart rate (HR) recovery (HRR) reflects autonomic activity and predicts cardiovascular events. The aim of this study was to assess HRR in patients with myocardial bridge (MB).

Methods: Medical recordings of 93 patients with MB and appropriate age, compared to 78 sex-matched healthy subjects were analyzed. MB was diagnosed via coronary computed tomography angiography after a positive exercise stress test (EST). HRR indices were calculated by subtracting $1^{\text {st }}$ (HRR1), $2^{\text {nd }}$ (HRR2) and $3^{\text {rd }}$ (HRR3) minute HR from the maximal HR during EST.

Results: HRR1 (30.2 \pm 13.3 bpm vs. $35.8 \pm 10.4$ bpm, $p=0.001)$ and HRR2 (52.3 $\pm 13.3 \mathrm{bpm}$ vs. $57.1 \pm 11.6 \mathrm{bpm}, p=0.013)$ were lower in patients with MB. In addition, HRR1 was lower in patients with left anterior descending $(L A D) M B$ than non- $L A D M B(28.5 \pm 13.2$ vs. $37.1 \pm 11.4$, $p=0.013)$. Presence of $M B$, deep $M B, L A D M B$ and multi-vessel $M B$ were predictors of $H R R 1$ $(p<0.01$ for all). In a multivariate analysis, LAD MB was the only significant independent predictor of HRR1 $(\beta=-8.524, p=0.009)$.

Conclusions: Patients with MB have impairment in HRR indices which is more pronounced among patients with LAD MB. Cardiac autonomic dysfunction in MB might be due to recurrent myocardial ischemia. (Cardiol J 2016; 23, 5: 524-531)
\end{abstract}

Key words: autonomic functions, coronary arteries, heart rate recovery, myocardial bridge

\section{Introduction}

The myocardial bridging (MB) is an anomaly characterized by a typical intramyocardial route of a segment of one of the major coronary arteries [1,2].
In such patients, there is a temporary systolic coronary arterial luminal narrowing. The frequency of $\mathrm{MB}$ reported in angiographic studies varies from $0.5-15 \%$, however, the frequency reported was $80 \%$ in the autopsy series [1-4]. It could be diag-

Address for correspondence: Sercan Okutucu, MD, Memorial Ankara Hospital, Department of Cardiology, Cankaya/Ankara, P.O: 06520, Turkey, tel: +90 3122536666 (ext.4207), mobile: +90 532 3314198, fax: +90 3122536623 ,

e-mail: sercanokutucu@yahoo.com

Received: 16.01.2016

Accepted: 23.06.2016 
nosed on conventional angiography, intravascular ultrasonography and coronary computed tomography angiography (CCTA) $[5,6]$. The incidence of MB increased because of the widespread use of these techniques $[1,2]$. Patients with MB usually have good prognosis, but some cases associated with myocardial ischemia, infarction, coronary spasm, arrhythmias, and sudden death have been reported [1,2].

Heart rate (HR) recovery (HRR) is a readily obtainable, relatively inexpensive and very simple diagnostic and prognostic tool that reflects the cardiac autonomic functions, which clinicians can apply in various clinical settings [7]. HRR indices show the rate of decline in the HR after the cessation of exercise test and it is defined as HR difference between the maximal HR on exercise and the HR during recovery phase. HRR after graded exercise reflects autonomic activity and predicts cardiovascular events and mortality not only in cardiovascular system disorders but also in various systemic disorders [8, 9]. As part of a regular routine exercise stress test (EST), HRR provides a risk assessment tool of a prognostic value for the clinicians $[7,10]$.

Although the HRR indices were assessed in many forms of coronary artery diseases (CAD) [10-12], cardiac autonomic functions and HRR indices have not been previously assessed in patients with MB [7]. Therefore, we aimed to assess HRR in patients with MB and compare with control subjects.

\section{Methods}

\section{Study population}

A total of 93 patients aged $32.2 \pm 8.6$ years (77 males) with MB were retrospectively selected from our hospital cardiology and radiology database between January 2012 to October 2015. Patients with concomitant atherosclerotic heart disease, diabetes mellitus, chronic renal failure, chronic liver disease, neurologic diseases, structural heart disease, pulmonary hypertension, rhythm abnormalities, chronotropic incompetence, drug use which has effect on autonomic system, and smoking habit were excluded from the study. The study population was relatively young and naïve to medication. Among patients with MB, 4 subjects reported using nonsteroidal anti-inflammatory drugs (NSAIDs) and 2 subjects were on proton pump inhibitor (PPI) treatment. Among healthy volunteers, 4 were on NSAID and 3 were on PPI treatment. All patients with MB had a positive EST and presence of MB was diagnosed by CCTA. The reason for abnormal positive EST was the development of horizontal or downsloping ST depression ( $\geq 0.5 \mathrm{~mm}$ at the $\mathrm{J}$-point in $\geq 2$ contiguous leads) in 73 patients, exertion-limiting symptoms in 16 and hypotensive response in 4 patients. We compared the clinical and exercise test data of these patients with 78 volunteers $(31.8 \pm 9.7$ years, 67 males) matched for age and sex. Echocardiographic examination and measurements were performed based on the criteria of the American Society of Echocardiography [13].

\section{Computerized tomography scanning protocol}

All studies were performed on 256-slice scanner (Siemens SOMATOM Definition Flash, Siemens Healthcare, Forchheim, Germany). A bolus injection of $80-100 \mathrm{~mL}$ non-ionic contrast medium iopromide (Ultravist 370, Bayer HealthCare Pharmaceuticals, Montville, New Jersey) or iopamidol (Iopamiro 370, Bracco, Milano, Italy) was intravenously injected, followed by a bolus of $40-50 \mathrm{~mL}$ of normal saline. The region of interest was manually placed on the left atrium. After the injection, an automatic triggering technique was activated with a preset delay (usually $5-8 \mathrm{~s}$ ) by the presence of a 150 Hounsfield Unit (HU) at the left atrium. If the resting HR was consistently above $70 \mathrm{bpm}$, the patient was prepared with metoprolol (5-15 mg, Beloc $5 \mathrm{mg} / 1 \mathrm{~mL}$, AstraZeneca, Istanbul, Turkey) before the examination. In each patient, the depth of the MB was analyzed and classified as superficial or deep with respect to the depth $(\leq 1$ or $>1 \mathrm{~mm}$ ) of the tunneled segment. Prospective electrocardiography (ECG) gating technique was used with CCTA that uses forward-looking prediction of $\mathrm{R}$ wave timing, step-and-shoot non-spiral acquisition with no table motion during imaging, and unique cone beam reconstruction [14].

\section{Treadmill exercise testing}

A treadmill exercise testing was conducted in all patients using modified Bruce protocol. Twelve lead ECG (Mason-Likar modification) was recorded at $25 \mathrm{~mm} / \mathrm{s}$ paper speed. All patients achieved an exercise time of more than $6 \mathrm{~min}$, and a final HR of at least $85 \%$ of age-predicted maximal HR response. After achieving peak workload, all patients spent 3-min recovery without cool-down period. HRR indices were calculated by subtracting $1^{\text {st }}$, $2^{\text {nd }}, 3^{\text {rd }}$ minute HR from the maximal HR obtained during stress testing and designated as HRR1, HRR2, and HRR3. Exercise capacity was measured in metabolic equivalent levels (METs) at peak 


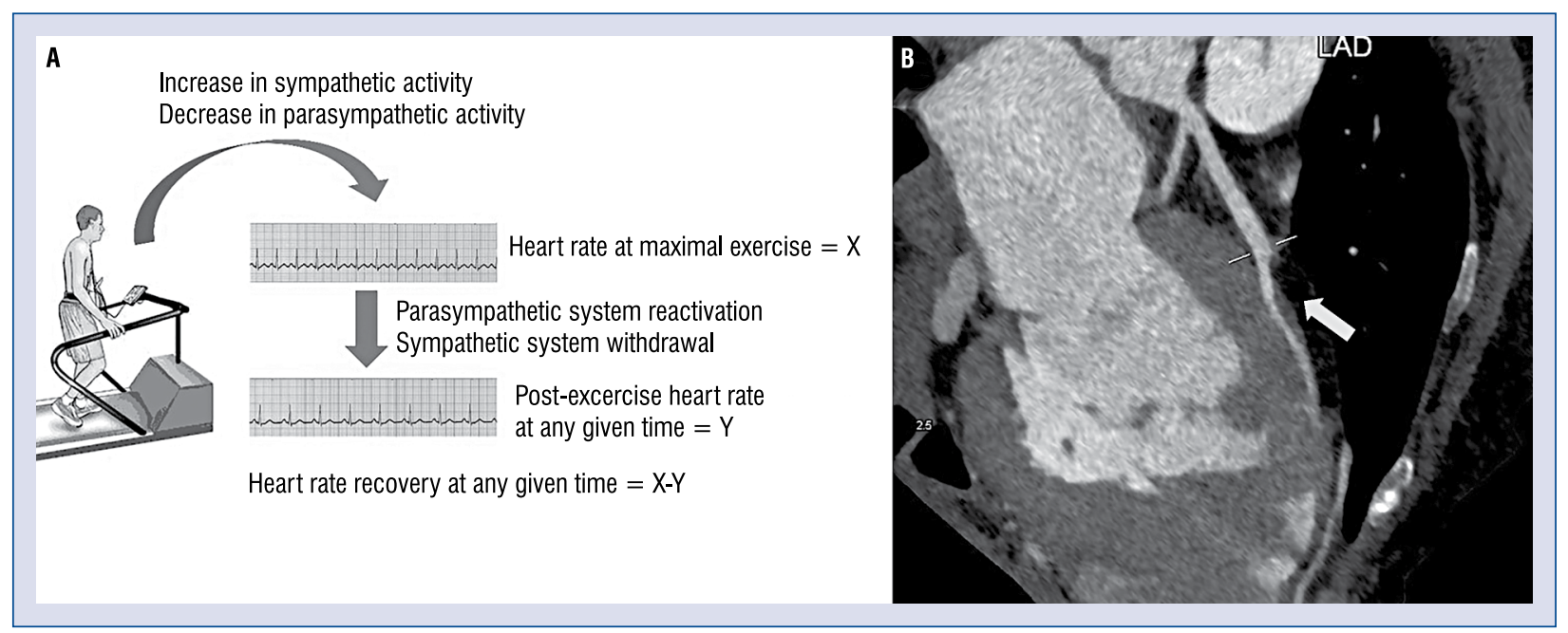

Figure 1. A. Measurement of heart rate recovery and (B) myocardial bridging causing luminal compression (arrow); LAD - left anterior descending artery.

Table 1. Demographic characteristics and clinical parameters of the groups.

\begin{tabular}{lccc}
\hline Variable & Patients with MB $(\mathbf{n}=\mathbf{9 3})$ & Control group $(\mathbf{n}=\mathbf{7 8})$ & $\mathbf{P}$ \\
\hline Age [years] & $32.2 \pm 8.6$ & $31.8 \pm 9.7$ & 0.785 \\
Gender (male, n) & 77 & 67 & 0.676 \\
SBP [mm Hg] & $126.1 \pm 16.0$ & $124.2 \pm 15.2$ & 0.440 \\
DBP [mm Hg] & $69.8 \pm 9.9$ & $69.3 \pm 8.9$ & 0.739 \\
Basal heart rate [bpm] & $72.9 \pm 6.1$ & $73.2 \pm 5.7$ & 0.681 \\
LV end-diastolic diameter [mm] & $45.4 \pm 3.0$ & $45.8 \pm 2.9$ & 0.430 \\
LV end-systolic diameter [mm] & $28.3 \pm 2.3$ & $27.8 \pm 2.4$ & 0.185 \\
LV ejection fraction [\%] & $62.0 \pm 3.0$ & $62.8 \pm 2.8$ & 0.234 \\
\hline
\end{tabular}

Numeric variables with a normal distribution were presented as the mean \pm standard deviation; DBP — diastolic blood pressure; LV — left ventricular; $\mathrm{MB}$ - myocardial bridge; SBP — systolic blood pressure

exercise. Duration of the treadmill exercise test, maximal HR, systolic blood pressure at rest (SBP) and at maximum exercise $\left(\mathrm{SBP}_{\mathrm{ME}}\right)$; diastolic blood pressure at rest (DBP) and at maximum exercise $\left(\mathrm{DBP}_{\mathrm{ME}}\right)$ were recorded. Measurement of HRR and $\mathrm{MB}$ causing coronary artery compression are shown in Figure 1.

\section{Statistical analysis}

Distribution of data was assessed using a one-sample Kolmogorov-Smirnov test. Data are demonstrated as mean \pm standard deviation (SD) for normally distributed continuous variables and frequencies for categorical variables. For numerical variables, an independent sample t-test and Mann-Whitney U test were used for intergroup comparisons. A $\chi^{2}$ test and Fischer's exact $\chi^{2}$ test were used for comparisons of categorical variables.
Univariate linear regression analysis was performed to evaluate the effects of variables on HRR. Predictors obtained from univariate analysis were further checked with multivariate linear regression analysis. Statistical analysis of the data was conducted using SPSS 15 (SPSS Inc., Chicago, IL, USA) and 2-tailed p-value $<0.05$ was considered statistically significant.

\section{Results}

Patients with $\mathrm{MB}$ and control group were similar with respect to age, gender and left ventricular ejection fraction. Demographic characteristics and clinical parameters of the groups are summarized in Table 1 .

Mean HRR1 $(30.2 \pm 13.3$ bpm vs. $35.8 \pm 10.4$ bpm, $\mathrm{p}=0.001)$ and HRR2 values $(52.3 \pm 13.3$ vs. $57.1 \pm 11.6, p=0.013)$ were significantly lower in 


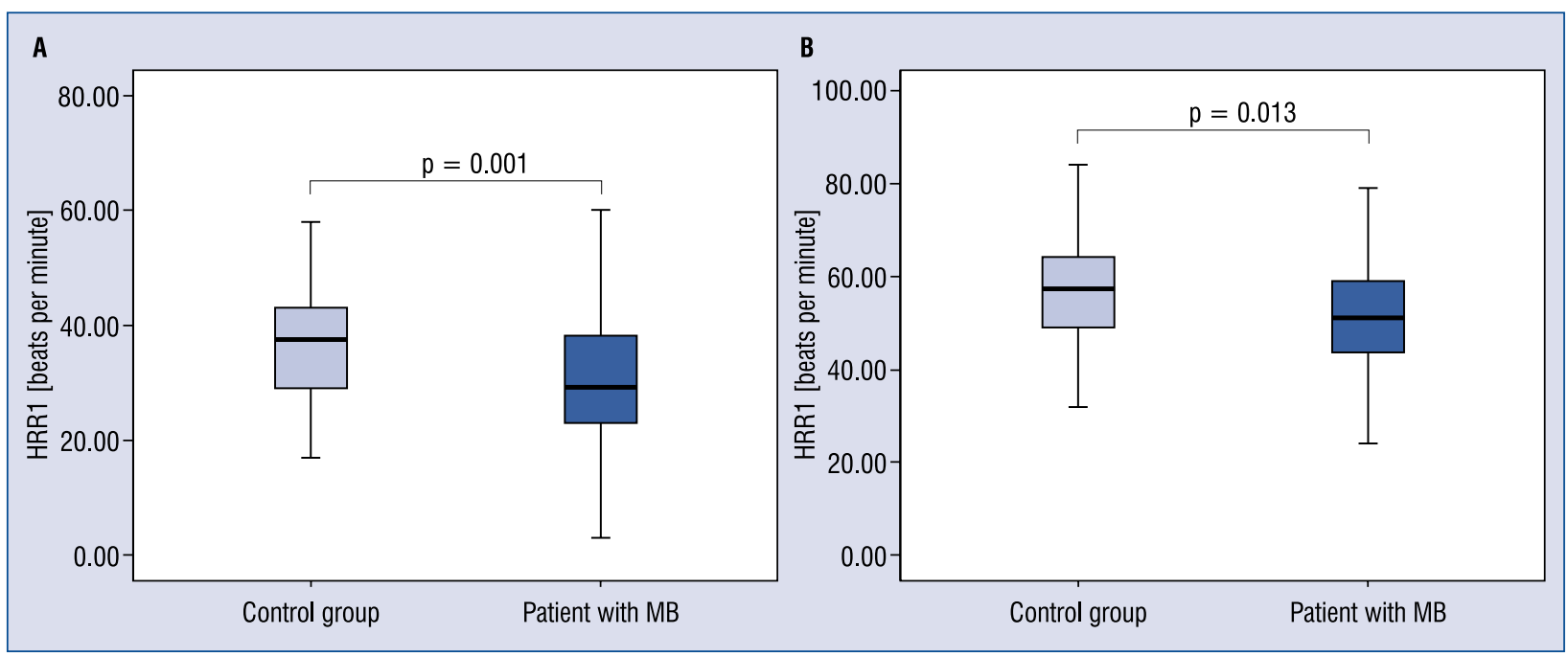

Figure 2. Comparison of (A) heart rate recovery at $1^{\text {st }}$ minute (HRR1) and (B) heart rate recovery at $2^{\text {nd }}$ minute (HRR2) in patients with myocardial bridge (MB) and control group.

Table 2. Treadmill exercise test parameters of the groups.

\begin{tabular}{lccc}
\hline Variable & Patients with MB $(\mathbf{n}=\mathbf{9 3})$ & Control group $(\mathbf{n}=\mathbf{7 8})$ & $\mathbf{P}$ \\
\hline Duration of exercise $[\mathrm{min}]$ & $9.8 \pm 1.9$ & $9.5 \pm 1.7$ & 0.266 \\
Peak exercise capacity [METs] & $12.0 \pm 2.0$ & $11.9 \pm 2.0$ & 0.703 \\
Maximal heart rate [bpm] & $174.1 \pm 12.6$ & $175.4 \pm 9.5$ & 0.462 \\
SBP $_{\text {ME }}[\mathrm{mm} \mathrm{Hg}]$ & $154.9 \pm 21.1$ & $152.8 \pm 12.8$ & 0.421 \\
DBP $_{\text {ME }}[\mathrm{mm} \mathrm{Hg}]$ & $80.5 \pm 14.4$ & $82.2 \pm 16.2$ & 0.467 \\
HRR1 $[\mathrm{bpm}]$ & $30.2 \pm 13.3$ & $36.3 \pm 10.5$ & 0.001 \\
HRR2 $[\mathrm{bpm}]$ & $52.3 \pm 13.3$ & $57.1 \pm 11.6$ & 0.013 \\
HRR3 $[\mathrm{bpm}]$ & $57.7 \pm 13.9$ & $60.2 \pm 12.9$ & 0.221 \\
\hline
\end{tabular}

Numeric variables with a normal distribution were presented as the mean \pm standard deviation; HRR1-3 - heart rate recovery at $1^{\text {st }}, 2^{\text {nd }}$ or $3^{\text {rd }}$ minute; $\mathrm{DBP}_{\mathrm{ME}}$ - diastolic blood pressure at maximum exercise; MB - myocardial bridge; METs - metabolic equivalent levels; $\mathrm{SBP}_{\mathrm{ME}}-$ systolic blood pressure at maximum exercise

patients with MB than the control group (Fig. 2). The duration of the treadmill exercise test, peak exercise capacity, maximal $\mathrm{HR}, \mathrm{SBP}_{\mathrm{ME}}, \mathrm{DBP}_{\mathrm{ME}}$, and HRR3 were similar in patients with MB and control group (Table 2).

Among patients with $\mathrm{MB}, 71(76.3 \%)$ cases had $1 \mathrm{MB} ; 15(16.1 \%)$ cases had $2 \mathrm{MB}$ and 7 (7.6\%) cases had $>2 \mathrm{MB}$. In 35 (37.6\%) of 93 subjects, the MBs were localized superficially, and $58(62.4 \%)$ of 93 subjects were deeply in myocardium. In patients with $\mathrm{MB}$, left anterior descending (LAD) coronary artery was most common site ( 75 patients, $80.6 \%$ ). Most frequently involved site of LAD was mid portion $(\mathrm{n}=57,76.0 \%)$ followed by proximal $(\mathrm{n}=13$, $17.3 \%)$ and distal portion $(\mathrm{n}=11,14.7 \%)$. Other localizations were circumflex coronary artery in
$19(20.4 \%)$, obtuse marginal artery in $16(17.2 \%)$, diagonal branch in $7(7.5 \%)$ and right coronary artery in $5(5.4 \%)$ patients. HRR1 was lower in patients with LAD MB than patients with non-LAD MB $(28.5 \pm 13.2$ vs. $37.1 \pm 11.4, p=0.013)$ (Fig. 3$)$. HRR indices were similar between superficial and deep MB $(31.6 \pm 14.6$ vs. $29.3 \pm 12.5, \mathrm{p}=0.417$ for HRR1; $54.0 \pm 13.8$ vs. $51.3 \pm 13.0, \mathrm{p}=0.349$ for HRR2; $59.8 \pm 14.3$ vs. $56.5 \pm 13.6, \mathrm{p}=0.283$ for HRR3) sub-groups.

Effects of age, gender, basal HR, maximal HR, $\mathrm{SBP}_{\mathrm{ME}}, \mathrm{DBP}_{\mathrm{ME}}$, exercise time, exercise capacity, presence of $\mathrm{MB}$, depth of $\mathrm{MB}, \mathrm{MB}$ localization and multiple MB on HRR indices were examined in a univariate linear regression analysis separately. Presence of MB $(\beta=-6.108, \mathrm{p}=0.001)$, deep MB 


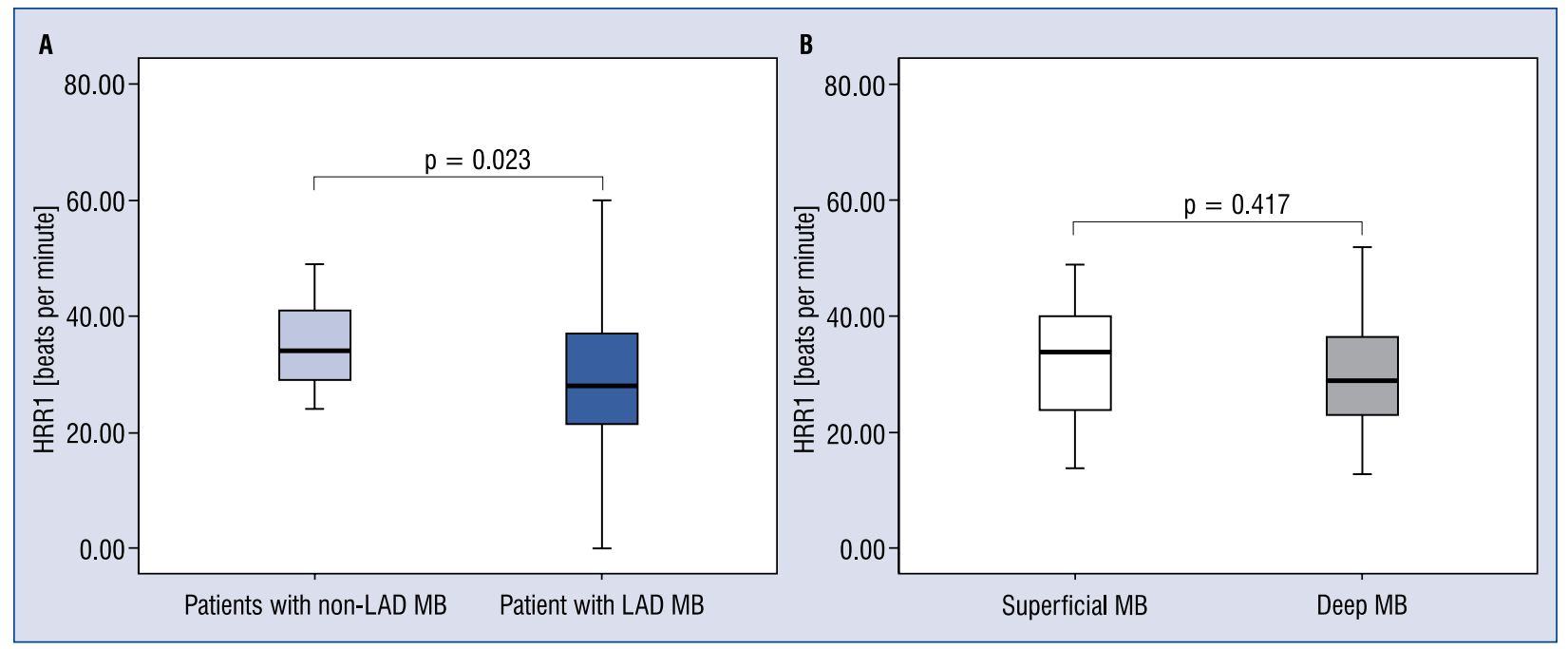

Figure 3. Comparison of heart rate recovery at $1^{\text {st }}$ minute (HRR1) $(A)$ in patients with non-left anterior descending (LAD) myocardial bridge (MB) and LAD MB, (B) in patients with superficial MB and deep MB.

Table 3. Effects of selected variables on heart rate recovery at 1-minute (HRR1) in multivariate linear regression.

\begin{tabular}{lcccc}
\hline Variable & $\beta$ & \multicolumn{2}{c}{$95 \%$ confidence interval for $\beta$} & P \\
\cline { 3 - 4 } & & Lower bound & Upper bound & \\
\hline Presence of MB & 2.202 & -6.720 & 11.124 & 0.627 \\
Deep MB & -0.714 & -5.984 & 4.557 & 0.790 \\
LAD MB & -8.524 & -14.934 & -2.113 & 0.009 \\
Multiple MB & -0.749 & -4.840 & 3.341 & 0.718 \\
\hline
\end{tabular}

LAD — left anterior descending; $M B$ - myocardial bridge

$(\beta=-5.561, \mathrm{p}=0.005), \mathrm{LAD} \mathrm{MB}(\beta=-7.934$, $\mathrm{p}=0.001)$, andmulti-vesselMB $(\beta=-3.203, \mathrm{p}=0.007)$ were found to be significant predictors of HRR1. These significant variables were further analyzed in multivariate linear regression model (Table 3). Presence of MB on LAD was found to be only significant independent predictor of HRR1 $(\beta=-8.524 ; 95 \% \mathrm{CI}-14.934$ to $-2.113 ; \mathrm{p}=0.009)$.

\section{Discussion}

To the best of our knowledge, our study is the first to suggest that the presence of lower HRR indices in patients with MB. This finding was more prominent among MB patients with LAD MB. Importantly, MB localized on LAD was a single independent predictor of lower HRR1 after graded exercise.

Myocardial bridging is defined as a segment of a major coronary artery course through the myocardium that normally has an epicardial course $[15,16]$. In the last decade, it was shown that myocardial bridging can be very well depicted by CCTA which has increased the interest in this anomaly $[3,17]$. Myocardial bridging is usually asymptomatic but might lead to myocardial ischemia, infarction, coronary spasm, arrhythmias, and sudden death [18-20].

An attenuated HRR, which is defined as an inadequate decline in HR immediately after exercise, reflects reduced parasympathetic nervous system activity [21,22]. The decline in HR during recovery is principally due to a reactivation of parasympathetic nervous system, mostly in the early recovery period [23]. Kannankeril et al. [10] demonstrated that sympathetic withdrawal also contributes significantly to early HRR, as evidenced by brisk HRR even after atropine administration at maximal exercise. Based on these findings, they suggested that abnormal HRR might be attributable to a defect 
in sympathetic withdrawal and parasympathetic reactivation or both of them. As these changes correlate with increased risk of death, it was hypothesized that an attenuated HRR would similarly predict an increased risk of death [24].

Abnormalities on EST, such as ST segment depression, typical chest pain or hypotensive response to exercise are often used to detect presence of CAD. As part of EST, HRR provides a risk assessment tool which gives prognostic information $[7,10]$. Impaired HRR indices are also associated with a higher susceptibility for atherosclerosis. Multiple studies showed abnormal HRR to be an independent risk factor for all-cause mortality at 5 years [25]. Previous studies of patients referred for coronary angiography for suspected CAD have suggested an association between blunted HRR and higher atherosclerotic burden [21]. Furthermore, blunted HRR has been observed to be associated with several risk factors for $\mathrm{CAD}$ [26]. Although the HRR indices were assessed in many forms of CAD, HRR indices have not been previously assessed in patients with MB. Therefore, our study is the first study to assess cardiac autonomic functions via HRR in patients with MB.

In patients with $\mathrm{MB}$, there is a temporarily increased pressure gradient on the proximal segments of MB due to systolic narrowing of coronary artery. Endothelial dysfunction, increased $\mathrm{Ca}^{+2}$ elements on the proximal arterial segment of $\mathrm{MB}$ and increased pressure gradient cause recurrent coronary vasospasm leading to myocardial ischemia and cardiovascular events [27]. In the current study, we found that patients with MB have lower HRR indices with respect to normal subjects. This finding might be due to the silent or symptomatic myocardial ischemia. It has been shown that patients with silent myocardial ischemia have significantly more impairment in HRR indices than patients without myocardial ischemia [28].

In this study, we found a more profound HRR index impairment in patients with LAD MB. These findings imply the presence of cardiac autonomic dysfunction in patients with $\mathrm{MB}$, especially in $\mathrm{LAD}$ involvement. Presence of MB particularly localized on LAD was the only independent predictor of lower HRR1 after graded exercise. This finding might be explained by the extent of ischemia and major epicardial coronary involvement. In accordance with this finding, Ghaffari et al. [11] found that abnormal HRR was independently correlated with the extent of ischemia and major epicardial coronary involvement in patients with CAD [11].
Relation between autonomic nervous system and MB has not been specifically investigated before. In an animal study, Tangkawattana et al. [29] documented that musculature MB was directly innervated by the middle cervical ganglion and left vagal nerve indicating that any imbalance in favor of sympathetic activity inevitably the musculature of MB. Consequently, MB may be abnormally influenced by sympathetic over activity either due to abovementioned pathophysiological mechanisms or due to imbalance in autonomic nervous system innervating musculature of MB. Documentation of sympathetic hyperactivity in patients with MB may be not only an advantage but also a rationale for the management of patients with MB. Initially prescribing regular exercising to these patients may alleviate the frequency of recurrent angina and also the threshold for it. Since exercise training favorably alters the influence of sympathetic neural activity on cardiovascular system by increasing the vagal tonus [30]. It may reduce the sympathetic drive leading the sign and symptoms related with MB. It was reported that beta-blocker therapy has improved the clinical sign and symptoms of patients with MB [2,31]. It has been proposed that beta-blocker therapy provides the clinical improvement by reducing the systolic compression and negative inotropic effect [32]. It was documented that hypertensive patients with silent ischemia had higher plasma noradrenaline levels than those without ischemia and metoprolol reduced the frequency of ischemic periods probably neutralizing the sympathetic activity [33]. Therefore, the presence of $\mathrm{MB}$, which may cause either silent or obvious ischemia, may probably be associated with increased noradrenalin levels and increased sympathetic activity. Furthermore, an ancillary treatment modality of partial sympathetic denervation which has been claimed to prevent recurrent vasospastic activity in variant angina may currently be investigated in the treatment of recurrent angina, myocardial ischemia, and infarction leading cardiovascular events in patients with MB [34].

In this study, HRR indices of patients with MB and control subjects seem to be in normal range taking into account cut-off values, which were established earlier in different clinical settings $[7,25]$. These results might be explained by influence of underlying pathology, mode of exercise, body posture during exercise, and recovery period may influence the peak HR at maximal exercise and HRR response [7, 35]. Therefore, it should be 
kept in mind that the exercise protocol of choice inevitably alters the decline in HR after exercise. Besides, there were statistically significant difference between patients with MB and matched healthy volunteers.

\section{Conclusions}

In conclusion, HRR after graded exercise is one of the commonly used technique which reflects autonomic functions and predicts cardiovascular events and mortality not only in cardiovascular system disorders but also in various systemic disorders. Patients with MB had lower HRR indices with respect to normal healthy subjects. The HRR index impairment was more profound in patients with LAD MB. These findings suggest the presence of cardiac autonomic dysfunction in patients with $\mathrm{MB}$, especially in LAD involvement. Having considered the significance of HRR, patients with LAD MB need to be followed for adverse cardiovascular outcomes.

\section{Conflict of interest: None declared}

\section{References}

1. Kalaria VG, Koradia N, Breall JA. Myocardial bridge: A clinical review. Catheter Cardiovasc Interv, 2002; 57: 552-556. doi: 10.1002/ccd.10219.

2. Lee MS, Chen CH. Myocardial bridging: An up-to-date review. J Invasive Cardiol, 2015; 27: 521-528.

3. Aparci M, Okutucu S, Ozturk C, Balta S, Isilak Z. Utility of multidetector tomography angiography with equivocal exercise stress test in acute coronary syndrome. Emerg Med Australas, 2014; 26: 517-518. doi: 10.1111/1742-6723.12269.

4. Mohlenkamp S, Erbel R. Myocardial bridging/angiographic prevalence of myocardial bridging. Anadolu Kardiyol Derg, 2006; 6: 195-196; author reply: 6.

5. Evangelista A, Pelliccia F, Arrivi A, Gaudio C. 320-slice CT and myocardial bridge. BMJ Case Rep, 2011; 2011. doi: 10.1136/ bcr.05.2011.4200.

6. Li JJ, Shang ZL, Yao M et al. Angiographic prevalence of myocardial bridging in a defined very large number of Chinese patients with chest pain. Chin Med J (Engl), 2008; 121: 405-408.

7. Okutucu S, Karakulak UN, Aytemir K, Oto A. Heart rate recovery: A practical clinical indicator of abnormal cardiac autonomic function. Expert Rev Cardiovasc Ther, 2011; 9: 1417-1430. doi: 10.1586/erc.11.149.

8. Imai K, Sato H, Hori M et al. Vagally mediated heart rate recovery after exercise is accelerated in athletes but blunted in patients with chronic heart failure. J Am Coll Cardiol, 1994; 24: 1529-1535.

9. Pierpont GL, Stolpman DR, Gornick CC. Heart rate recovery post-exercise as an index of parasympathetic activity. J Auton Nerv Syst, 2000; 80: 169-174.

10. Kannankeril PJ, Le FK, Kadish AH, Goldberger JJ. Parasympathetic effects on heart rate recovery after exercise. J Investig Med, 2004; 52: 394-401.
11. Ghaffari S, Kazemi B, Aliakbarzadeh P. Abnormal heart rate recovery after exercise predicts coronary artery disease severity. Cardiol J, 2011; 18: 47-54.

12. Morshedi-Meibodi A, Larson MG, Levy D, O'Donnell CJ, Vasan RS. Heart rate recovery after treadmill exercise testing and risk of cardiovascular disease events (The Framingham Heart Study). Am J Cardiol, 2002; 90: 848-852.

13. Lang RM, Badano LP, Mor-Avi V et al. Recommendations for cardiac chamber quantification by echocardiography in adults: an update from the American Society of Echocardiography and the European Association of Cardiovascular Imaging. Eur Heart J Cardiovasc Imaging, 2015; 16: 233-270. doi: 10.1093/ehjci/jev014.

14. Hsieh J, Londt J, Vass M, Li J, Tang X, Okerlund D. Step-andshoot data acquisition and reconstruction for cardiac x-ray computed tomography. Med Phys, 2006; 33: 4236-4248.

15. Yu M, Zhou L, Chen T, Yi K, Zeng C, Tan X. Myocardia ischemia associated with a myocardial bridge with no significant atherosclerotic stenosis. BMC Cardiovasc Disord, 2015; 15: 165. doi: 10.1186/s12872-015-0158-2.

16. Ishii T, Ishikawa Y, Akasaka Y. Myocardial bridge as a structure of "double-edged sword" for the coronary artery. Ann Vasc Dis, 2014; 7: 99-108. doi: 10.3400/avd.ra.14-00037.

17. Nieman K, Coenen A. Myocardial enhancement defects on CT angiograms: Promising prognosticator or water under the bridge? J Am Coll Cardiol Cardiovasc Imaging, 2015; 8: 695-696. doi: 10.1016/j.jcmg.2015.02.011.

18. Vales L, Kanei Y, Fox J. Coronary artery occlusion and myocardial infarction caused by vasospasm within a myocardial bridge. J Invasive Cardiol, 2010; 22: E67-E69.

19. Li ZF, Yang SG, Ge JB. Acute myocardial infarction due to myocardial bridge. Chin Med J (Engl), 2012; 125: 3589-3590.

20. Li JJ. Is myocardial bridging a bridge connecting to cardiovascular events? Chin Med J (Engl), 2010; 123: 964-968.

21. Vivekananthan DP, Blackstone EH, Pothier CE, Lauer MS. Heart rate recovery after exercise is a predictor of mortality, independent of the angiographic severity of coronary disease. J Am Coll Cardiol, 2003; 42: 831-838. doi: S0735109703008337.

22. Arena R, Guazzi M, Myers J, Peberdy MA. Prognostic value of heart rate recovery in patients with heart failure. Am Heart J, 2006; 151: 851 e7-13. doi: 10.1016/j.ahj.2005.09.012.

23. Tang YD, Dewland TA, Wencker D, Katz SD. Post-exercise heart rate recovery independently predicts mortality risk in patients with chronic heart failure. J Card Fail, 2009; 15: 850-855. doi: 10.1016/j.cardfail.2009.06.437.

24. Goldberger JJ, Cain ME, Hohnloser SH et al. American Heart Association/american College of Cardiology Foundation/heart Rhythm Society scientific statement on noninvasive risk stratification techniques for identifying patients at risk for sudden cardiac death: A scientific statement from the American Heart Association Council on Clinical Cardiology Committee on Electrocardiography and Arrhythmias and Council on Epidemiology and Prevention. Heart Rhythm, 2008; 5: e1-e21. doi: 10.1016/j. hrthm.2008.05.031.

25. Nishime EO, Cole CR, Blackstone EH, Pashkow FJ, Lauer MS. Heart rate recovery and treadmill exercise score as predictors of mortality in patients referred for exercise ECG. JAMA, 2000; 284: 1392-1398.

26. Kizilbash MA, Carnethon MR, Chan C, Jacobs DR, Sidney S, Liu K. The temporal relationship between heart rate recovery immediately after exercise and the metabolic syndrome: The CARDIA study. Eur Heart J, 2006; 27: 1592-1596. doi: 10.1093/ /eurheartj/ehl043. 
27. Aparci M, Yalcin M, Isilak Z. Accumulation of $\mathrm{Ca}(+2)$ at arterial segments proximal to myocardial bridging may influence the vascular dynamics of coronary artery. Int J Cardiol, 2016; 202: 782-783. doi: 10.1016/j.ijcard.2015.10.061.

28. Yamada T, Yoshitama T, Makino K, Lee T, Saeki F. Heart rate recovery after exercise is a predictor of silent myocardial ischemia in patients with type 2 diabetes. Diabetes Care, 2011; 34: 724-726. doi: 10.2337/dc10-1424.

29. Tangkawattana P, Muto M, Nakayama T, Karkoura A, Yamano S, Yamaguchi M. Prevalence, vasculature, and innervation of myocardial bridges in dogs. Am J Vet Res, 1997; 58: 1209-1215.

30. Leosco D, Parisi V, Femminella GD et al. Effects of exercise training on cardiovascular adrenergic system. Front Physiol, 2013; 4: 348. doi: 10.3389/fphys.2013.00348.

31. Schwarz ER, Klues HG, vom Dahl J, Klein I, Krebs W, Hanrath P. Functional, angiographic and intracoronary Doppler flow char- acteristics in symptomatic patients with myocardial bridging: Effect of short-term intravenous beta-blocker medication. J Am Coll Cardiol, 1996; 27: 1637-1645.

32. Nair CK, Dang B, Heintz MH, Sketch MH. Myocardial bridges: Effect of propranolol on systolic compression. Can J Cardiol, 1986; 2: 218-221.

33. Lee DD, Kimura S, DeQuattro V. Noradrenergic activity and silent ischaemia in hypertensive patients with stable angina: Effect of metoprolol. Lancet, 1989; 1: 403-406.

34. Betriu A, Pomar JL, Bourassa MG, Grondin CM. Influence of partial sympathetic denervation on the results of myocardial revascularization in variant angina. Am J Cardiol, 1983; 51: 661-667.

35. Okutucu S, Kabakci G, Deveci OS et al. Relationship between exercise heart rate recovery and circadian blood pressure pattern. J Clin Hypertens (Greenwich), 2010; 12: 407-413. doi: 10.1111/j.1751-7176.2010.00279.x. 